\title{
An Ultra-Wideband Band Pass Filter using Metal Insulator Metal Waveguide for Nanoscale Applications
}

\author{
Surendra Kumar Bitra \\ Department of Electronics and Communication Engineering \\ Koneru Lakshamaiah Education Foundation \\ Vaddeswaram, Guntur District, Andhra Pradesh, India \\ bitrasurendrakumar@gmail.com
}

\author{
Sridhar Miriyala \\ Department of Electronics and Communication Engineering \\ Koneru Lakshamaiah Education Foundation \\ Vaddeswaram, Guntur District, Andhra Pradesh, India \\ Sridhar.m@kluniversity.in
}

\begin{abstract}
A T-stub Square Ring Resonator (SRR) based UltraWide Band (UWB) Band Pass Filter (BPF) is studied and investigated in this paper. The proposed filter is based on coupled feed line connected to the T-stub SRR. Ultra-wideband characteristics can be realized by adjusting the T-stub lengths and coupling the gaps between both sides of waveguides and SRR. The characteristics of the T-stub SRR show that the miniaturized UWB BPF can be operated at THz frequencies. The proposed UWB filter is simulated and analyzed using the Finite Differential Time Domain (FDTD) solver-based Computer Simulation Technology (CST) studio suite. The resonance conditions are explained and the transmission performance of the filter agrees with the simulated and theoretical calculations. The proposed filter is best suitable for Electronic-Plasmonic Integrated Circuits (EPICs).
\end{abstract}

Keywords-Metal-Insulator-Metal (MIM); ring resonator; coupled lines; Finite Differential Time Domain (FDTD); UltraWide Band (UWB); T-stub

\section{INTRODUCTION}

The light incident on a metal-insulator region produces a high-speed electromagnetic (EM) wave called Surface Plasmon Polarity (SPP) [1]. SPPs are known for overcoming the diffraction limit of the light at nanoscale wavelengths. Due to the low loss and high confinement of light at nanoscale range the Metal-Insulator-Metal (MIM) waveguide has been proposed by several researchers [2-4]. The subwavelength optical components that have been proposed when using MIM waveguide are reported in [5-9]. Several researchers worked on theories and experimentations of stub resonators like disk resonators [10], teeth-shaped resonators [11], rectangular [12], triangular [13], square [14], and circular [15] ring resonators. A Band Pass Filter (BPF) with symmetric side couple nano-disk resonators is analyzed for $1310 \mathrm{~nm}$ and $1550 \mathrm{~nm}$ in [16]. A concurrent dual band BPF is designed using slot waveguides and the performance is carried out at the resonant frequencies of $1300 \mathrm{~nm}$ and $1600 \mathrm{~nm}$ respectively in [17]. A tunable stepped impedance ring resonator for dual band BPF is designed and analysed at $\mathrm{O}$ and $\mathrm{L}$ bands in [18]. However, most of the investigations are carried out in single-, dual- and triple-band of operation for BPF. A limited work is carried out in Ultra-Wide
Band (UWB) BPF where the bandwidth is limited, and the size of UWB filters is more. The optical bands used for transmission are O-band (1260-1360nm), E-band (1360$1460 \mathrm{~nm})$, S-band (1460-1530nm), C-band (1530-1565nm), and the L-band $(1565-1625 \mathrm{~nm})$. The ring resonators are a fundamental component used in the Electronic-Photonic Integrated Circuits (EPICs). MIM and Insulator Metal-Insulator (IMI) are important geometries used in plasmonic waveguides. IMI waveguides carry light in longer propagation distances than MIM waveguides due to their cladding layers, but the MIM structures have higher confinement. Due to the high confinement and moderate propagation lengths, the MIM structures are efficiently used by EPICs. Authors in [19] designed a two-dimensional structure consisting of a MIM waveguide with a ring resonator, and two resonant peaks observed. This work is a further extension of the SRR for UWB applications.

Most of the existing resonators have symmetrical shapes. In this work, a nanoplasmonic T-stub loaded Square Ring Resonator (SRR) at optical bands is proposed. Most of the existing work was carried out on the design of single and dualoperating bands. In our proposed work, more than one band of frequencies are considered simultaneously (Ultra-Wide BandUWB).

\section{T-STUB SRR FILTER DESIGN}

SRR filter for dual band and SRR with stubs for triple band applications have been designed and their performance was investigated in our previous work [20]. In this paper, a T-stub SRR filter is designed which is suitable for UWB applications with compact dimensions. The methodology of the proposed filter design is illustrated with the flowchart shown in Figure 1. Firstly, the T-Stub SRR is designed using the Computer Simulation Technology (CST) studio suite. Simulations are carried out under Perfect Magnetic Layer (PML) boundary conditions for getting suitable transmission and reflection parameters. Figure 2 represents the proposed UWB filter designed using SRR. Four T-stubs are included at the four sides of the square ring representing the proposed T-stub SRR. The blue color in Figure 2 represents the metal and the gray color 
indicates the insulator. Silver is used as metal and silica is used as an insulator. The silver metal permittivity is calculated using the Drude model [21], i.e. $\varepsilon(m)=1-\omega_{p}^{2} / \omega\left(\omega+i \gamma_{p}^{2}\right)$, where $\omega_{p}\left(=1.38 \times 10^{16}\right)$ is the plasma frequency and $\gamma_{p}\left(=2.73 \times 10^{13}\right)$ is the collision frequency. $\mathrm{SiO}_{2}\left(\varepsilon_{i}=2.5\right)$ is used as an insulator and its permittivity is considered constant [21].

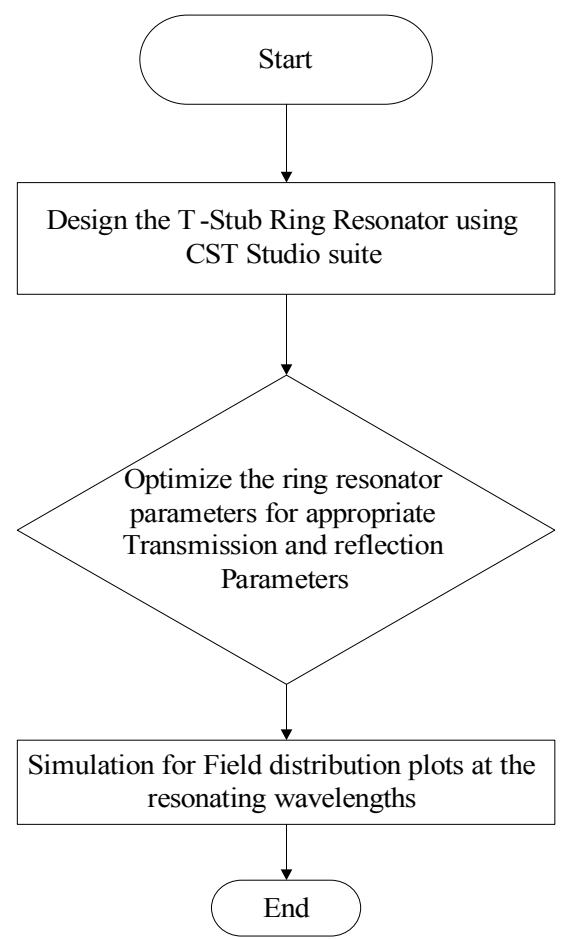

Fig. 1. Methodology of proposed T-stub ring resonator.

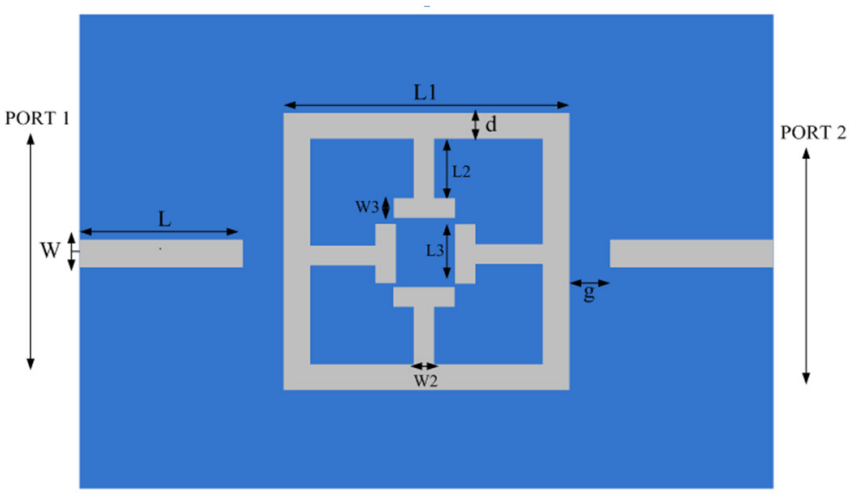

Fig. 2. SRR based UWB filter.

Two waveguides are situated on both sides of the T-stub SRR filter. The dimensions of the waveguide, T-stubs and SRR are tabulated in Table I. The basic properties of MIM waveguide like refractive index $\left(N_{e f f}\right)$, propagation length $(P L)$ of SPP and calculated dispersion relation are defined in [17]. The proposed T-stub SRR filter is designed and analyzed using a commercially available Finite Differential Time Domain
(FDTD) solver-based CST studio suite. PML boundary conditions with mesh size of $5 \mathrm{~nm} \times 5 \mathrm{~nm}$ were used in simulations. Two power monitors were included at the two ports of the filter (port 1 and port 2) to measure the incident power $\left(P_{\text {in }}\right)$ and transmitted power $\left(P_{\text {out }}\right)$. At port 1 (input port), part of the EM waves is reflected at the interface of the MIM waveguide and the remaining part of the EM waves is coupled to the SRR cavity with a small gap $(g)$. The transmitting and reflecting EM waves in the SRR form standing waves and these are again coupled to the output port (port 2). The rectangular ring resonance condition is given by [12]:

$$
\lambda_{m}=\frac{\lambda_{0}}{\left(\beta_{g} / \beta_{0}\right)}=\frac{l_{1}+l_{2}}{m}
$$

where $m=0,1,2,3 \ldots, \quad\left(\beta_{g} / \beta_{0}\right)$ is the normalized propagation constant, and $\lambda_{0}$ is the fundamental wavelength. The overall length of the SRR can be calculated from $L=N \lambda_{g}=N\left(\lambda_{0} / \operatorname{Re}\left(n_{\text {eff }}\right)\right)$ where $N=1,2,3 \ldots, \lambda_{g}$ is the guided wavelength and $n_{\text {eff }}$ is the effective refractive index of the MIM waveguide.

TABLE I. DIMENSIONS OF SRR BASED UWB FILTER

\begin{tabular}{|c|c|c|}
\hline S. No & Parameter & Dimensions (nm) \\
\hline $\mathbf{1}$ & $L$ & 100 \\
\hline $\mathbf{2}$ & $W$ & 60 \\
\hline $\mathbf{3}$ & $L 1$ & 1100 \\
\hline $\mathbf{4}$ & $d$ & 60 \\
\hline $\mathbf{5}$ & $L 2$ & 295 \\
\hline $\mathbf{6}$ & $W 2$ & 50 \\
\hline $\mathbf{7}$ & $L 3$ & 275 \\
\hline $\mathbf{8}$ & $W 3$ & 55 \\
\hline $\mathbf{9}$ & $g$ & 3 \\
\hline
\end{tabular}

Simulations were carried out for rectangular ring resonator observed the dual band operation [11]. Including the T-stubs on the four sides of the SRR forms the proposed UWB structure and operated $\mathrm{O}, \mathrm{E}, \mathrm{S}$, and $\mathrm{C}$ bands simultaneously. The proposed filter is very useful for EPICs. The fabrication of MIM waveguide can be done by film deposition and photolithography techniques.

\section{RESULTS AND DISCUSSION}

The reflection and transmission characteristics of the T-stub SRR are represented in Figure 3. The simulations were carried in the FDTD solver-based CST microwave studio suite under PML boundary conditions and mesh settings of $5 \mathrm{~nm} \times 5 \mathrm{~nm}$. The simulation results of Figure 3(a)-(b) clearly show the filter operating in the $\mathrm{O}, \mathrm{E}, \mathrm{S}$, and $\mathrm{C}$ bands simultaneously. The transmission characteristics of the proposed T-stub SRR depends on the electromagnetic resonance of the SRR. The transmission and reflection characteristics are represented in Figure 3(a) for varying $L 3$, from 290 to 300 with a step size of $5 \mathrm{~nm}$. For increasing $L 3$ the covering UWB is moved right as shown in Figure 3(a). The optimum length $L 3$ is $295 \mathrm{~nm}$. The bandwidth observed is $\sim 300 \mathrm{~nm}$ by the T-stub SRR. By varying the optimum width $W 3$ of the T-slot from $50 \mathrm{~nm}$ to $60 \mathrm{~nm}$ with a step size of $5 \mathrm{~nm}$, the UWB spectrum covers higher wavelengths i.e from $1240 \mathrm{~nm}$ to $1570 \mathrm{~nm}$. However, the 
reflection coefficient is near to the $-10 \mathrm{~dB}$ range bound. The optimimum width for the T-stub is $55 \mathrm{~nm}$. The other parameters $L, W, W 2, d$, and $L 2$ have negligible effect on the UWB spectrum. The varied $W 3$ reflection and Transmission coefficient is represented in the Figure 3(b). By varying the gap $g$ between the coupled waveguide and SRR, the operating UWB band shifts to the right (Figure 3(c)). The value of $g$ varies from 2 to $4 \mathrm{~nm}$ with a step size of $1 \mathrm{~nm}$. For the $2 \mathrm{~nm}$ gap, the reflection coefficient is above $-10 \mathrm{~dB}$ for wavelengths from $1360 \mathrm{~nm}$ to $1450 \mathrm{~nm}$, for $3 \mathrm{~nm}$ the $\mathrm{RC}$ is less than $-10 \mathrm{~dB}$ throughout the bands, and for $4 \mathrm{~nm}$ gap the bands are decreasing. Due to that, $3 \mathrm{~nm}$ is considered the optimum gap of the proposed UWB filter. The bandwidth of the proposed UWB observed from the simulation results is $\sim 300 \mathrm{~nm}$. The symmetrical MIM waveguides are located on both sides of the T-stub SRR, the electromagnetic wave in the T-stub SRR will form the stable resonance observed at three wavelengths shown in Figure 3.

(a)

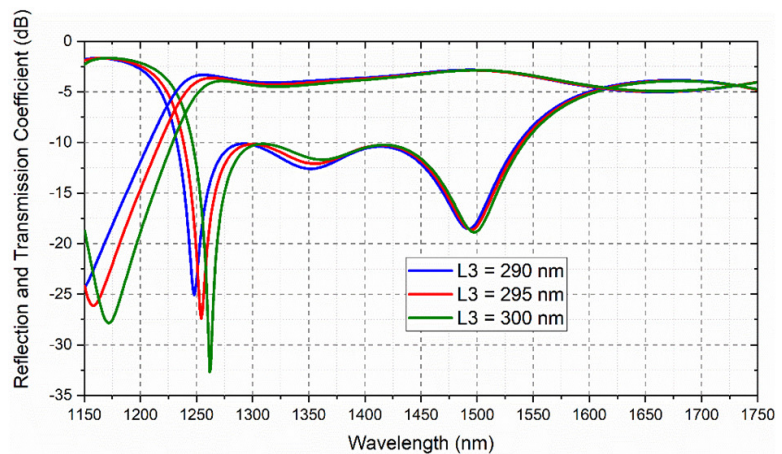

(b)

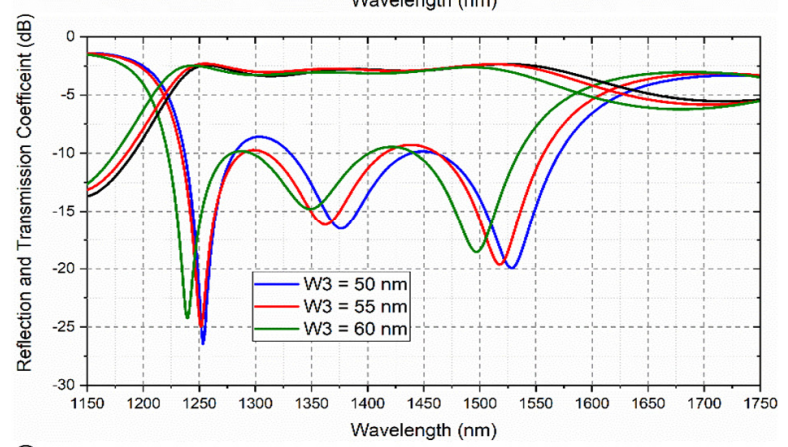

(c)

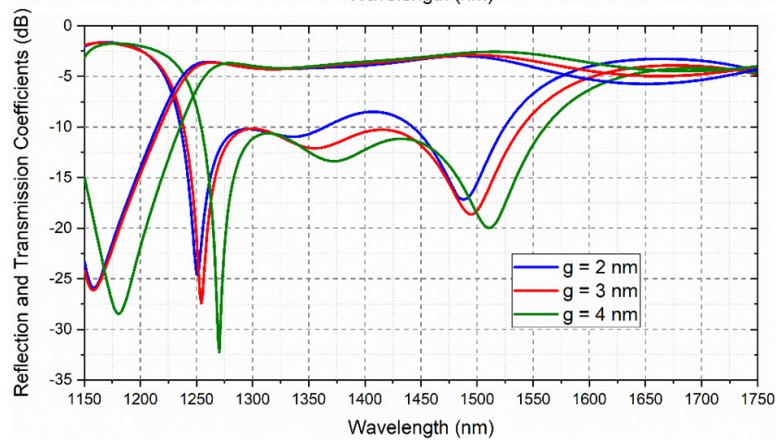

Fig. 3. Reflection and transmission coefficients for: (a) varying $L 3$,(b) varying $W 3$, and (c) varying gap $g$ between the coupled feed and the SRR.

The proposed UWB filter is compared with existing filter models in terms of parameters like operating wavelength,
Extinction Ratio (ER), Bandwidth (BW), Area $(L \times W)$ occupied by the filter, and application bands. The proposed filter provides an improved bandwidth of $310 \mathrm{~nm}$ when compared with the bandwidth $(150 \mathrm{~nm})$ of the filter presented in [23]. Even though the bandwidth is low for the proposed filter in comparison with the filter of [4] (320nm), it operates in four operating bands $(\mathrm{O}, \mathrm{S}, \mathrm{C}$, and $\mathrm{E})$.

(a)

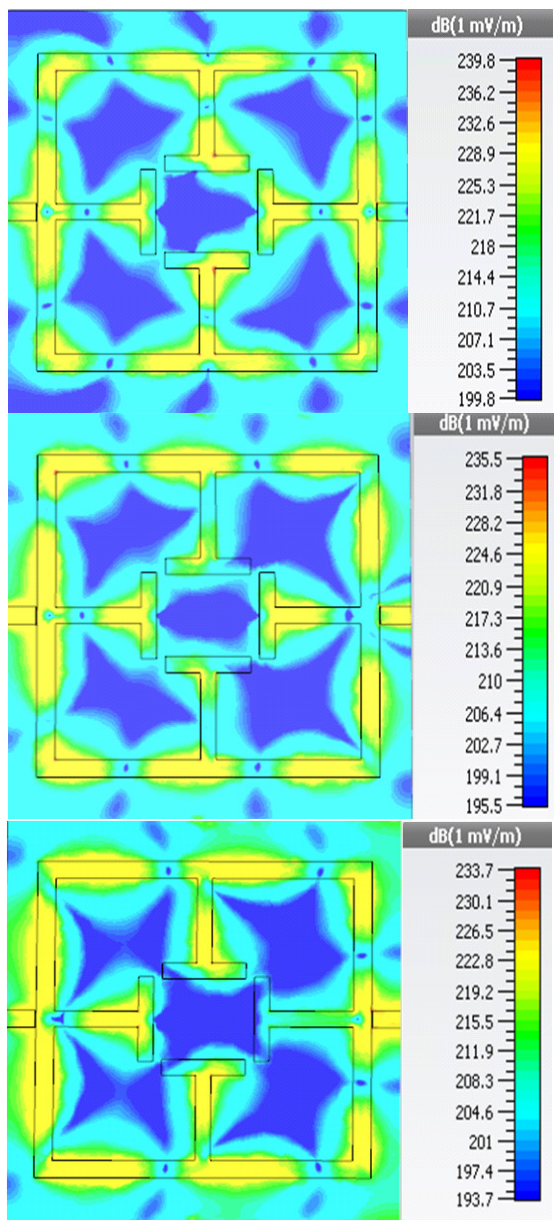

Fig. 4. Field distributions: (a) $1264.94 \mathrm{~nm}(237 \mathrm{THz})$, (b) $1362.69 \mathrm{~nm}$ (220THz), and (c) $1495.9 \mathrm{~nm}(200.4 \mathrm{THz})$.

The proposed UWB T-stub SRR is giving better performance in terms of BW, filter size, and application bands as can be seen in Table II.

TABLE II. COMPARISON OF THE PROPOSED FILTER WITH EXISTING MODELS

\begin{tabular}{|c|c|c|c|c|c|}
\hline $\begin{array}{c}\text { Filter } \\
\text { models }\end{array}$ & $\begin{array}{c}\text { Operating } \\
\text { wavelength (nm) }\end{array}$ & ER & $\begin{array}{c}\text { BW } \\
(\mathbf{n m})\end{array}$ & $\begin{array}{c}\boldsymbol{L} \times \boldsymbol{W} \\
\left(\mathbf{n m}^{2}\right)\end{array}$ & $\begin{array}{c}\text { Application } \\
\text { bands }\end{array}$ \\
\hline $\begin{array}{c}\text { BPF based on } \\
\text { ARR [4] }\end{array}$ & $1190-1510$ & $\sim 38$ & 320 & $1100 \times 1620$ & $\begin{array}{c}\mathrm{O}, \mathrm{S}, \mathrm{C} \text { and } \\
\text { partial E }\end{array}$ \\
\hline $\begin{array}{c}\text { BPF based on } \\
\text { U-shaped RR } \\
{[23]}\end{array}$ & $1280-1440$ & $\begin{array}{c}\sim 26 . \\
5\end{array}$ & 150 & $1120 \times 3232$ & $\mathrm{O}, \mathrm{S}$ \\
\hline $\begin{array}{c}\text { Proposed } \\
\text { filter based } \\
\text { on SRR }\end{array}$ & $1240-1550$ & $\begin{array}{c}\sim 28 . \\
1\end{array}$ & 310 & $1200 \times 1506$ & $\mathrm{O}, \mathrm{S}, \mathrm{C}$, and E \\
\hline
\end{tabular}


The energy of the SPP wave at UWB wavelengths shows the stronger coupling of the T-stub SRR filter at $1264.94 \mathrm{~nm}$ $(237 \mathrm{THz}), 1362.69 \mathrm{~nm}(220 \mathrm{THz})$, and $1495.9 \mathrm{~nm}(200.4 \mathrm{THz})$ wavelengths as shown in Figure 3. According to the simulation, the odd mode analysis has given better results than the even mode analysis of the T-stub SRR filter.

\section{CONCLUSION}

In this paper, the T-stub SRR filter for ultra-wide band applications based on transmission characteristics was studied. The coupled line MIM plasmonic structure is composed of two straight waveguides and the T-stub SRR. Simulations were carried out using the FDTD based CST studio suite. The simulation results show that the T-stub SRR filter gives better performance in $\mathrm{O}, \mathrm{E}, \mathrm{S}$, and $\mathrm{C}$ optical bands. The transmission is slightly smaller at bigger wavelengths when comparing with the transmission at smaller wavelengths. The proposed T-stub SRR is optimized for the parameters of $L 3$ (length of the stub) and gap $g$ between the MIM waveguide and SRR. The ER for the proposed T-Stub UWB filter is $\sim 28.1$ at $1252 \mathrm{~nm}$ and $\sim 17.5$ at $1492 \mathrm{~nm}$ respectively. The bandwidth for the UWB band is $310 \mathrm{~nm}$. The optimized parameters also show that the proposed filter is best suitable for optical UWB applications. A strong EM coupling was observed at the MIM waveguides and SRR throughout the UWB range. The proposed UWB is best suitable for EPICs for transmitting high speed data. These structures are fabricated using lithographic techniques.

\section{REFERENCES}

[1] W. $\mathrm{Mu}$ and J. B. Ketterson, "Long-range surface plasmon polaritons propagating on a dielectric waveguide support," Optics Letters, vol. 36, no. 23, pp. 4713-4715, Dec. 2011, https://doi.org/10.1364/OL.36. 004713.

[2] W. Liu, R. Zhong, J. Zhou, Y. Zhang, M. Hu, and S. Liu, "Investigations on a nano-scale periodical waveguide structure taking surface plasmon polaritons into consideration," Journal of Physics D-applied Physics - J PHYS-D-APPL PHYS, vol. 45, May 2012, https://doi.org/10.1088/0022$3727 / 45 / 20 / 205104$

[3] K. M. Krishna, M. Z. U. Rahman, and S. Y. Fathima, "A New Plasmonic Broadband Branch-Line Coupler for Nanoscale Wireless Links," IEEE Photonics Technology Letters, vol. 29, no. 18, pp. 1568-1571, Sep. 2017, https://doi.org/10.1109/LPT.2017.2737036.

[4] M. Z. U. Rahman, K. M. Krishna, K. K. Reddy, M. V. Babu, S. S. Mirza, and S. Y. Fathima, "Ultra-Wide-Band Band-Pass Filters Using Plasmonic MIM Waveguide-Based Ring Resonators," IEEE Photonics Technology Letters, vol. 30, no. 19, pp. 1715-1718, Oct. 2018, https://doi.org/10.1109/LPT.2018.2866966.

[5] M. Vishwanath, H. Khan, and K. Thirupathaiah, "Concurrent dual band filters using plasmonic MIM waveguide ring resonator," ARPN Journal of Engineering and Applied Sciences, vol. 13, no. 5, pp. 1813-1818, Mar. 2018.

[6] M. Vishwanath and H. Khan, "Systematic analysis of T-junctions using plasmonic MIM waveguide," Journal of Advanced Research in Dynamical and Control Systems, vol. 9, no. 14, pp. 1862-1868, Dec. 2017.

[7] G. Veronis and S. Fan, "Modes of Subwavelength Plasmonic Slot Waveguides," Journal of Lightwave Technology, vol. 25, no. 9, pp. 2511-2521, Sep. 2007, https://doi.org/10.1109/JLT.2007.903544.

[8] V. Mittapalli and H. Khan, "Excitation Schemes of Plasmonic Angular Ring Resonator-Based Band-Pass Filters Using a MIM Waveguide," Photonics, vol. 6, no. 2, Jun. 2019, Art. no. 41, https://doi.org/10.3390/ photonics6020041

[9] M. Vishwanath and D. Habibullakhan, "Design and Simulation of Compact Band-Pass Filter Using Stub Loaded Plasmonic Mim
Waveguide," International Journal of Engineering \& Technology, vol. 7, no. 3.3, pp. 41-43, Jun. 2018, https://doi.org/10.14419/ijet.v7i3. 3.14482 .

[10] H. Lu, X. Liu, D. Mao, L. Wang, and Y. Gong, "Tunable band-pass plasmonic waveguide filters with nanodisk resonators," Optics Express, vol. 18, no. 17, pp. 17922-17927, Aug. 2010, https://doi.org/10.1364/ OE.18.017922.

[11] X. Lin and X. Huang, "Numerical modeling of a teeth-shaped nanoplasmonic waveguide filter," Journal of the Optical Society of America B, vol. 26, no. 7, pp. 1263-1268, Jul. 2009, https://doi.org/ 10.1364/JOSAB.26.001263.

[12] B. Yun, G. Hu, and Y. Cui, "Theoretical analysis of a nanoscale plasmonic filter based on a rectangular metal-insulator-metal waveguide," Journal of Physics D: Applied Physics, vol. 43, no. 38, p. 385102, Sep. 2010, https://doi.org/10.1088/0022-3727/43/38/385102.

[13] G.-Y. Oh, D.-G. Kim, S. H. Kim, H. C. Ki, T. U. Kim, and Y.-W. Choi, "Integrated Refractometric Sensor Utilizing a Triangular Ring Resonator Combined With SPR," IEEE Photonics Technology Letters, vol. 26, no. 21, pp. 2189-2192, Nov. 2014, https://doi.org/10.1109/LPT.2014. 2349975.

[14] J. Liu, G. Fang, H. Zhao, Y. Zhang, and S. Liu, "Plasmon flow control at gap waveguide junctions using square ring resonators," Journal of Physics D: Applied Physics, vol. 43, no. 5, Jan. 2010, Art. no. 055103, https://doi.org/10.1088/0022-3727/43/5/055103.

[15] A. Setayesh, S. R. Mirnaziry, and M. S. Abrishamian, "Numerical Investigation of Tunable Band-pass $\backslash$ band-stop Plasmonic Filters with Hollow-core Circular Ring Resonator," Journal of the Optical Society of Korea, vol. 15, no. 1, pp. 82-89, Mar. 2011.

[16] S. Shi et al., "Enhanced plasmonic band-pass filter with symmetric dual side-coupled nanodisk resonators," Journal of Applied Physics, vol. 118, no. 14, Oct. 2015, Art. no. 143103, https://doi.org/10.1063/1.4932668.

[17] K. Thirupathaiah, N. P. Pathak, and V. Rastogi, "Concurrent Dual Band Filters Using Plasmonic Slot Waveguide," IEEE Photonics Technology Letters, vol. 25, no. 22, pp. 2217-2220, Nov. 2013, https://doi.org/ 10.1109/LPT.2013.2283880.

[18] H. Lu, G. Wang, and X. Liu, "Manipulation of light in MIM plasmonic waveguide systems," Chinese Science Bulletin, vol. 58, no. 30, pp. 3607-3616, Oct. 2013, https://doi.org/10.1007/s11434-013-5989-6.

[19] S. Bitra and S. Miriyala, "Concurrent Dual and Triple Band Square Ring Resonator Base-Band Filter using Metal-Insulator-Metal for Plasmonic Applications," Applied Computational Electromagnetics Society Journal, vol. 35, no. 10, Dec. 2020, https://doi.org/10.47037/2020. ACES.J.351010.

[20] S. A. Maier, Plasmonics: Fundamentals and Applications. New York, NY, USA: Springer, 2007.

[21] P. B. Johnson and R. W. Christy, "Optical Constants of the Noble Metals," Physical Review B, vol. 6, no. 12, pp. 4370-4379, Dec. 1972, https://doi.org/10.1103/PhysRevB.6.4370.

[22] S. K. Bitra and M. Sridhar, "Design of Nanoscale Square Ring Resonator Band-Pass Filter Using Metal-Insulator-Metal," in Microelectronics, Electromagnetics and Telecommunications, Singapore, 2021, pp. 659-664, https://doi.org/10.1007/978-981-153828-5_68.

[23] P. Osman, P. V. Sridevi, and K. V. S. N. Raju, "Design of MetalInsulator-Metal based Plasmonic Ultra-Wide Band U-Shaped resonators based Band-Pass Filter," International Journal for Research in Applied Science and Engineering Technology, vol. 7, no. 5, pp. 3737-3719, May 2019, https://doi.org/10.22214/ijraset.2019.5615. 